\title{
AVALIAÇÃO DA PRESENÇA DOS FATORES DE RISCO CARDIOVASCULAR EM ESTUDANTES UNIVERSITÁRIOS DE PAÍSES LUSÓFONOS*
}

\author{
Tibelle Freitas Mauricio ${ }^{1}$, Rafaella Pessoa Moreira², Edmara Chaves Costa ${ }^{3}$, Francisco Mardones \\ dos Santos Bernardo ${ }^{4}$, Paula Alves de Lima ${ }^{5}$, Bonifácio de Jesus Viegas ${ }^{4}$
}

\begin{abstract}
RESUMO: Objetivo: avaliar a presença de fatores de risco cardiovascular em estudantes universitários de países lusófonos. Metodologia: estudo transversal, realizado de janeiro a julho de 2017, com 334 estudantes de uma universidade pública federal no estado do Ceará, com idade igual ou superior a 18 anos, dos seguintes países: Brasil, Angola, Cabo Verde, Guiné-Bissau, Moçambique, São Tomé e Príncipe e Timor-Leste. Efetuou-se a análise estatística dos dados por meio do programa Epi Info versão 7.1.2.0. Resultados: dos 334 participantes, 75,7\% encontravam-se na faixa etária entre 18 e 24 anos, 56,9\% eram brasileiros, 51,8\% do sexo feminino, 47,3\% de cor parda e 47,6\% solteiros com parceria eventual. Quanto aos fatores de risco para doenças cardiovasculares, destacaram-se a história familiar de doenças cardiovasculares $(81,1 \%)$, o sedentarismo $(44,3 \%)$ e o uso do álcool $(36,2 \%)$. Conclusão: a identificação dos principais fatores de risco modificáveis e hereditários nos estudantes universitários poderão nortear ações voltadas para a promoção da saúde cardiovascular.
\end{abstract}

DESCRITORES: Risco; Doenças Cardiovasculares; Estudantes; Países em Desenvolvimento.

\section{EVALUATION OF THE PRESENCE OF CARDIOVASCULAR RISK FACTORS IN UNIVERSITY STUDENTS FROM PORTUGUESE-SPEAKING COUNTRIES}

ABSTRACT: Objective: to evaluate the presence of cardiovascular risk factors in university students from Portuguese-speaking countries. Methodology: cross-sectional study, carried out from January to July 2017, with 334 students from a federal public university in the state of Ceará, aged 18 years and over, from the following countries: Brazil, Angola, Cape Verde, Guinea-Bissau, Mozambique, Sao Tome and Principe and East Timor. Data was analyzed with the Epi Info version 7.1.2.0 statistical software. Results: of the 334 participants, 75.7\% were aged 18-24 years old, 56.9\% were Brazilian, $51.8 \%$ were female, $47.3 \%$ were brown and $47.6 \%$ were single individuals with an occasional partner. Risk factors for cardiovascular diseases included a family history of cardiovascular disease $(81.1 \%)$, sedentary lifestyle $(44.3 \%)$ and alcohol use $(36.2 \%)$. Conclusion: identification of the main modifiable and hereditary risk factors in university students could guide actions aimed at promoting cardiovascular health.

KEYWORDS: Risk; Cardiovascular diseases; Students; Developing countries.

\section{EVALUACIÓN DE LA PRESENCIA DE LOS FACTORES DE RIESGO CARDIOVASCULAR EN ESTUDIANTES UNIVERSITARIOS DE PAÍSES LUSÓFONOS}

RESUMEN: Objetivo: evaluar la presencia de factores de riesgo cardiovascular en estudiantes universitarios de países lusófonos. Metodología: estudio transversal, realizado de enero a julio de 2017, con 334 estudiantes de una universidad pública federal en el estado de Ceará, con edad igual o superior a 18 años, de los siguientes países: Brasil, Angola, Cabo Verde, Guinea-Bissau, Mozambique, San Tomé y Príncipe y Timor-Leste. Se hizo análisis estadístico de los datos por medio del programa Epi Info versión 7.1.2.0. Resultados: de los 334 participantes, 75,7\% estaban en edad entre 18 y 24 años, 56,9\% eran brasileños, 51,8\% del sexo femenino, 47,3\% de color pardo y 47,6\% solteros con pareja eventual. Acerca de los factores de riesgo para enfermedades cardiovasculares, se destacaron la historia familiar de enfermedades cardiovasculares $(81,1 \%)$, el sedentarismo (44,3\%) y el uso de alcohol (36,2\%). Conclusión: la identificación de los principales factores de riesgo modificables y hereditarios en los estudiantes universitarios podrán nortear acciones de promoción de salud cardiovascular.

DESCRIPTORES: Riesgo; Enfermedades cardiovasculares; Estudiantes; Países en desarrollo.

\footnotetext{
"Artigo extraído da dissertação do mestrado intitulada: Diagnóstico de enfermagem: Risco de função cardiovascular prejudicada em estudantes universitários de países lusófonos. Universidade da Integração Internacional da Lusofonia Afro-Brasileira, 2017.

'Enfermeira. Mestre em Enfermagem pela Universidade da Integração Internacional da Lusofonia Afro-Brasileira, Programa de PósGraduação em Enfermagem. Enfermeira do Instituto Federal de Educação, Ciências e Tecnologia do Ceará. Redenção, CE, Brasil. ${ }^{2}$ Enfermeira. Doutora em Enfermagem. Docente da Universidade da Integração Internacional da Lusofonia Afro-Brasileira em Enfermagem. Redenção, CE, Brasil.

${ }^{3}$ Médica veterinária. Pós-doutora. Docente da Universidade da Integração Internacional da Lusofonia Afro-Brasileira, Programa de Pós-Graduação em Enfermagem. Redenção, CE, Brasil.

${ }^{4}$ Enfermeiro. Universidade da Integração Internacional da Lusofonia Afro-Brasileira, Instituto de Ciências da Saúde.Redenção, CE, Brasil.

${ }^{5}$ Enfermeira. Universidade da Integração Internacional da Lusofonia Afro-Brasileira, Instituto de Ciências da Saúde.Redenção, CE, Brasil.
} 


\section{INTRODUÇÃO}

As doenças cardiovasculares (DCV) constituem a principal causa de óbito em todo o mundo. Somente no ano de 2015 foram responsáveis por aproximadamente 17,7 milhões de óbitos, representando 31\% de todas as mortes globais, das quais $82 \%$ se concentraram nos países de baixa e média renda, como o Brasil, países da África e da Ásia(1-2) que fazem parte dos países lusófonos, ou seja, países cujo povo fala a língua portuguesa.

No Brasil, as DCV são as principais causas de morte em homens e mulheres nas cinco regiões do país. Destaca-se que, no período de 1980 a 2012, a mortalidade por DCV se elevou na região Nordeste, ao contrário das demais regiões, onde se observou queda da mortalidade na população total ${ }^{(3)}$.

Essas doenças também representam a principal causa de morbidade e mortalidade por doenças nãotransmissíveis na África. Estima-se que, nessa região, os principais fatores de risco cardiovascular estejam associados à urbanização, à elevação do consumo de tabaco, a dietas pobres relacionadas a colesterol elevado e diabetes, bem como à inatividade física, levando ao sobrepeso ou à obesidade ${ }^{(4)}$.

Em síntese, os países subdenvolvidos ou em desenvolvimento, com condições precárias de vida, pelas condições socioeconômicas e, consequentemente, situações inadequadas de saúde, apresentam elevado número de pessoas acometidas por doenças cardiovasculares as quais podem se agravar devido ao diagnóstico tardio e à dificuldade de acesso ao tratamento de saúde.

Ressalta-se que as DCV são de origem multifatorial em que os fatores de risco modificáveis e não modificáveis participam de sua gênese. Essas doenças contêm variáveis que propiciam o aparecimento e o desenvolvimento das cardiopatias. Portanto, a devida monitoração contribui para a identificação dos sinais precursores que, ao serem modificados, poderão reduzir ou até mesmo reverter o processo evolutivo das disfunções ${ }^{(5)}$.

Quanto aos fatores de risco não modificáveis,há o fator hereditário, provável determinante para o surgimento das $D_{C}{ }^{(6)}$, sendo assim importante conhecer a história familiar para saber a qual doença a pessoa está mais predisposta. É válido salientar que a associação deste fator de risco com hábitos de vida poderá antecipar a manifestação de DCV.

Deste modo, a maioria das DCV pode ser evitada por estratégias que abranjam toda a população,por meio da abordagem aos fatores de risco comportamentais ${ }^{(1)}$.Para tanto, é imprescindível a identificação das pessoas expostas a esses fatores, de modo a sensibilizá-las a mudanças de comportamento, visto que muitos deles estão relacionados ao estilo de vida e são, assim, modificáveis. $E$, de fato, observa-se o desenvolvimento de múltiplas ações educativas na área da saúde pública voltadas para essa problemática ${ }^{(7)}$.

Desta feita, percebe-se a importância em identificar pessoas que tenham fatores de risco cardiovascular nos diferentes grupos e faixas etárias. Estima-se que mais da metade dos adultos jovens têm pelo menos um fator de risco para doença coronariana, o que eleva consideravelmente o risco de doença cardíaca ao longo da vida. Como diversos fatores de risco para doenças cardiovasculares surgem na adolescência e continuam na idade adulta, sugere-se que a prevenção inicie-se primordialmente na infância e adolescência ${ }^{(8)}$.

Neste âmbito, estudos constataram que adultos jovens universitários apresentam diversos fatores de risco para as $\mathrm{DCV}^{(8)}$. No entanto, apesar da elevada prevalência desses fatores de risco e dos sinais precoces de aterosclerose, o risco ainda é subestimado nesse grupo ${ }^{(9)}$. Portanto, torna-se imprescindível a investigação do risco cardiovascular nos estudantes universitários de diferentes nacionalidades, como é o caso do Brasil, de alguns países da África e Ásia, visto que a identificação precoce de fatores de risco para as DCV poderá favorecer uma intervenção efetiva, de modo a melhorar a qualidade de vida e prevenir futuros agravos nesse grupo.

Diante do exposto, esta pesquisa visou avaliar a presença de fatores de risco cardiovascular em estudantes universitários de países lusófonos. 


\section{METODOLOGIA}

Estudo descritivo, transversal, de abordagem quantitativa, realizado no período de janeiro a julho de 2017. Para a coleta de dados, optou-se por uma universidade pública federal cujos campi estão situados nos municípios de Acarape e Redenção, no estado do Ceará, e contemplam estudantes do Brasil e internacionais, de países lusófonos (Angola, Moçambique, Cabo Verde, Guiné-Bissau, São Tomé e Príncipe, Timor-Leste).

A população do estudo foi constituída por 2.496 estudantes universitários, de ambos os sexos, com idade igual ou superior a 18 anos, nascidos nos países supracitados, que estavam matriculados e frequentando regularmente os cursos do Programa de Graduação na modalidade presencial da universidade.

A amostra, calculada por meio da fórmula para determinação de amostra finita, contemplou 334 estudantes (Brasil: 190, Angola: 30, Cabo Verde: 11, Guiné-Bissau: 63, Moçambique: 11, São Tomé e Príncipe: 07 e Timor Leste: 22). Para os cálculos, consideraram-se: $N=2.496 ; p=0,25 ; \mathrm{q}=0,75 ; \mathrm{e}=$ $0,05(95 \%$ de confiança); $z=1,96$.

A coleta de dados foi efetuada por fonte primária (diretamente com os participantes do estudo), pela pesquisadora e estudantes voluntários do curso de enfermagem, os quais foram previamente submetidos a um treinamento de 4 horas, visando padronizar o método de coleta. A pesquisadora e os voluntários foram a campo convidar os estudantes universitários a participar do estudo, após explicação dos objetivos pretendidos e da metodologia a ser seguida. Depois da manifestação do interesse em participar da pesquisa, os estudantes assinaram o Termo de Consentimento Livre e Esclarecido.

Posteriormente, realizou-se a entrevista e o exame físico. Para a entrevista, aplicou-se um formulário elaborado pela pesquisadora com base nos fatores de risco cardiovascular, no qual constavam questões abertas e estruturadas, referentes à identificação do paciente, relacionando dados demográficos e sociais, informações sobre as condições de saúde, em especial da saúde cardiovascular e presença de fatores de risco para o surgimento de DCV. Depois da construção do formulário, este foi submetido ao pré-teste com 10 estudantes, e não foi necessário realizar alterações.

Em seguida, realizaram-se a coleta dos dados antropométricos (mensuração de peso, altura e circunferência abdominal) e a aferição da pressão arterial. Para a coleta de dados antropométricos, foram utilizados: balança antropométrica, devidamente testada e calibrada, com capacidade de 150 quilogramas (kg) e precisão de 100 gramas (g), com antropômetro acoplado e fita métrica inelástica. As medidas do peso foram feitas com o participante na posição ortostática, posicionado no centro da balança com os pés juntos e os braços estendidos ao longo do corpo. A estatura foi verificada por meio do antropômetro acoplado à balança. O participante ficou na posição ortostática, pés descalços e unidos, braços pendentes, com as mãos espalmadas sobre as coxas e queixo ereto.

O Índice de Massa Corporal foi obtido a partir da divisão do peso corporal em quilogramas pela estatura em metro elevada ao quadrado $\left(\mathrm{kg} / \mathrm{m}^{2}\right)$. Para a classificação dos valores, foi adotada a recomendação da Organização Mundial de Saúde (OMS) para avaliação de pessoas adultas e idosas: abaixo do normal (IMC $<18,5)$, normal $(18,5>I M C \leq 24,0)$, sobrepeso (IMC $\geq 25)$ e obesidade (IMC $\geq 30)^{(10)}$.

A medida da circunferência abdominal ocorreu com o paciente na posição ortostática. Utilizou-se, como ponto de referência, da metade da distância entre a crista ilíaca e o rebordo costal. Os valores considerados normais foram $<102 \mathrm{~cm}$ para os homens e $<88 \mathrm{~cm}$ para as mulheres ${ }^{(10)}$.

Para a verificação da pressão arterial, foram utilizados os seguintes equipamentos: esfigmomanômetros com manômetros aneroides, devidamente testados e calibrados, e estetoscópios duplos.

A interpretação dos valores da pressão arterial ocorreu por meio da comparação com tabelas propostas e reconhecidas como adequadas para a medida casual da pressão arterial em consultório, em indivíduos maiores de 18 anos, para classificação da Pressão Arterial Sistólica (PAS) mmHg e Pressão Arterial Diastólica (PAD) mmHg: normal ( $\leq 120$ e $\leq$ 80); Pré-hipertensão (121- 139 e 81- 89); Hipertensão estágio 1 (140 - 159 e 90 - 99); Hipertensão estágio 2 (160 - 179 e 100 - 109); Hipertensão estágio 3 ( $\geq 180$ e $\geq 110)$ e Hipertensão sistólica isolada $(\geq 140 \text { e }<90)^{(10)}$. 
Quanto à atividade física, definiu-se estilo de vida sedentário como a ausência de atividade física (inferior a 25 - 30 minutos/dia durante 3 dias/semana), e atividade física moderada a realização de atividade por mais de 30 minutos em, pelo menos, 3 dias por semana ${ }^{(11)}$.

No tocante à ingestão de bebida alcoólica, investigou-se o consumo e a frequência (diária, semanal, mensal ou esporádica). Definiu-se consumo abusivo de álcool, a ingesta de cinco ou mais doses de bebida alcoólica (homem) ou quatro ou mais doses (mulher) em uma única ocasião, pelo menos uma vez nos últimos 30 dias $^{(12)}$. Igualmente, questionou-se o uso de tabaco e o número de cigarros por dia. Para tanto, tomou-se como referência a Organização Mundial de Saúde ${ }^{(13)}$.

Os dados coletados foram tabulados em planilha eletrônica do Programa Excel for Windows ${ }^{\circledR} 2010$ e a análise estatística foi feita com o programa Epi Info versão 7.1.2.0. Aplicaram-se, inicialmente, procedimentos estatísticos descritivos, incluindo distribuição de frequências para as variáveis qualitativas e medidas de tendência central e variabilidade, adequados à síntese dos dados numéricos.

Respeitaram-se os princípios éticos da pesquisa científica preconizados pela Comissão Nacional de Ética em Pesquisa. O projeto foi aprovado pelo Comitê de Ética em Pesquisa da Universidade da Integração Internacional da Lusofonia Afro-Brasileira (Unilab), com o número do parecer: 1.873.108.

\section{RESULTADOS}

Os resultados são inicialmente apresentados na Tabela 1 de acordo com o perfil socio-demográfico dos participantes, contemplando: faixa etária, nacionalidade, sexo, cor autorreferida e estado civil. Por conseguinte, a Tabela 2 apresenta os fatores de risco cardiovascular identificados nos estudantes.

Tabela 1 - Perfil socio-demográfico dos estudantes universitários dos países lusófonos (n: 334). Redenção, CE, 2017. (continua)

\begin{tabular}{lcc}
\hline Variável & № & $\%$ \\
\hline Faixa Etária & 253 & 75,7 \\
\hline $18-24$ & 72 & 21,6 \\
$25-31$ & 4 & 1,2 \\
$32-38$ & 2 & 0,6 \\
$39-45$ & 1 & 0,3 \\
$46-52$ & 2 & 0,6 \\
\hline $53-59$ & & \\
\hline Nacionalidade & 190 & 56,9 \\
\hline Brasil & 30 & 9 \\
Angola & 11 & 3,3 \\
Cabo Verde & 63 & 18,9 \\
Guiné-Bissau & 11 & 3,3 \\
Moçambique & 7 & 2 \\
São Tomé e Príncipe & 22 & 6,6 \\
\hline Timor-Leste & & 51,8 \\
\hline Sexo & 173 & 48,2 \\
\hline Feminino & 161 & 9 \\
Masculino & & \\
\hline Cor autorreferida & 30 & \\
\hline Branca & & \\
\hline
\end{tabular}




\begin{tabular}{lcc} 
Preta & 144 & 43,1 \\
Amarela & 2 & 0,6 \\
Parda & 158 & 47,3 \\
\hline Estado Civil & & 47,6 \\
\hline Solteiro (a) com parceria eventual & 159 & 46,1 \\
Solteiro (a) com parceria fixa & 154 & 5,1 \\
Casado (a) ou em união consensual & 17 & 0,3 \\
Viúvo (a) & 1 & 0,9 \\
Divorciado (a) & 3 & 18 \\
\hline
\end{tabular}

Dos 334 participantes do estudo, 75,7\% (253) apresentavam faixa etária entre 18 e 24 anos, 56,9\% (190) eram brasileiros, 51,8\% (173) do sexo feminino, 47,3\% (158) de cor parda e 47,6\% (159) solteiros com parceria eventual.

Tabela 2 - Distribuição dos estudantes universitários conforme os fatores de risco para as doenças cardiovasculares (DCV) (n: 334). Redenção, CE, 2017

\begin{tabular}{|c|c|c|}
\hline Variável & № & $\%$ \\
\hline \multicolumn{3}{|c|}{ Tabagismo } \\
\hline Sim & 18 & 5,4 \\
\hline Não & 316 & 94,6 \\
\hline \multicolumn{3}{|c|}{ Consumo de álcool } \\
\hline Sim & 121 & 36,2 \\
\hline Não & 213 & 63,8 \\
\hline \multicolumn{3}{|c|}{ Sedentarismo } \\
\hline Sim & 148 & 44,3 \\
\hline Não & 186 & 55,7 \\
\hline \multicolumn{3}{|c|}{ Obesidade } \\
\hline Sim & 21 & 6,3 \\
\hline Não & 313 & 93,7 \\
\hline \multicolumn{3}{|c|}{ Hipertensão Arterial } \\
\hline Sim & 15 & 4,5 \\
\hline Não & 319 & 95,5 \\
\hline \multicolumn{3}{|c|}{ História Pessoal de DCV } \\
\hline Sim & 35 & 10,5 \\
\hline Não & 299 & 89,5 \\
\hline \multicolumn{3}{|c|}{ História Familiar de DCV } \\
\hline Sim & 271 & 81,1 \\
\hline Não & 63 & 18,9 \\
\hline \multicolumn{3}{|c|}{ Conhecimento sobre os fatores de risco } \\
\hline Sim & 226 & 67,7 \\
\hline Não & 108 & 32,3 \\
\hline
\end{tabular}

No tocante aos fatores de risco para DCV, destacaram-se: história familiar de doenças cardiovasculares, presente em 81,1\% (271) dos estudantes universitários; estilo de vida sedentário em 44,3\% (148) e uso de álcool por 36,2\% (121), conforme exposto na Tabela 2. 


\section{DISCUSSÃO}

O perfil dos estudantes em relação à faixa etária foi semelhante ao encontrado em outros estudos, realizados em instituições educacionais do Chile e do México, que também investigaram os fatores de risco cardiovascular em estudantes universitários. Na pesquisa realizada no Chile, a idade média dos estudantes do primeiro ano foi de 19,2 $\pm 1,8$ anos e dos estudantes do terceiro, de $21,7 \pm 2,5$. No estudo do México, a idade média foi de 20 anos, com variação de 16 a 27 anos $^{(6,14)}$. No que se refere à predominância do sexo feminino, a composição da amostra estudada se assemelha à de outros estudos que trabalharam com este grupo ${ }^{(15-16)}$.

Quanto aos fatores de risco, destacou-se a história familiar de DCV, tendo em vista que foi referida por $271(81,1 \%)$ estudantes, contemplando: hipertensão arterial sistêmica (HAS), diabetes, cardiopatias, dislipidemias, infarto agudo do miocárdio e acidente vascular encefálico. Pesquisa realizada com estudantes de uma universidade do México também investigou a história familiar de DCV, em que se destacou a hipertensão arterial $(45,8 \%)^{(14)}$.

Aponta-se que as doenças cardiovasculares hereditárias constituem um grupo de entidades com ampla diversidade clínica e genética. Pesquisas moleculares desenvolvidas em variadas populações comprovaram associação de cada uma dessas condições a centenas de mutações patogênicas distintas. É válido salientar que a variabilidade clínica dessas enfermidades pode igualmente ser elucidada por fatores epigenéticos e/ou pela interação com o ambiente ${ }^{(17)}$.

Logo, o conhecimento da história familiar de doenças é de fundamental relevância para o desenvolvimento de estratégias voltadas para prevenir e/ou retardar o aparecimento desses agravos, especialmente em adultos jovens, uma vez que constituem a faixa etária produtiva e, ao adoecerem poderão ter dificuldade em acessar e/ou permanecer no mercado de trabalho etambém aumentarão os gastos familiares com o tratamento.

Além disso, sabe-se que a prevenção dos riscos cardiovasculares desde a fase jovem, a qual coincide com o ingresso na vida universitária, é fundamental para chegar à fase senil com mais saúde e, consequentemente, melhor qualidade de vida.

Outro achado relevante foi o sedentarismo, presente em148 (44,3\%) participantes, cujo resultado assemelhou-se ao de outra pesquisa com estudantes, a qual sugeriu que a maior utilização do computador e a elevada ocupação do tempo, voltada para as atividades acadêmicas, interferem negativamente na prática de atividade física ${ }^{(18)}$.

No Brasil, constatou-se que, entre as 26 capitais brasileiras e o Distrito Federal, a frequência de adultos fisicamente inativos foi de $15,4 \%$, com frequência semelhante entre homens $(16,2 \%)$ e mulheres $(14,7 \%)$. Salienta-se que a porcentagem de pessoas fisicamente inativas foi mais elevada em adultos com menor escolaridade (até 8 anos de estudo) e em pessoas a partir de 65 anos, para ambos os sexos ${ }^{(12)}$.

Ressalta-se que a atividade física regular traz benefícios relevantes para a saúde, pois está diretamente relacionada com a redução dos fatores de risco cardiovascular, visto que melhora os níveis séricos de colesterol e lipoproteína de alta densidade (HDL-C), reduz o colesterol total (CT), a lipoproteína de baixa densidade (LDL-C), os triglicerídeos (TG), a pressão arterial e auxilia no controle glicêmico ${ }^{(19)}$. Além disso, aumenta a circulação neurológica, influenciando, assim, a aprendizagem e estimula a liberação de adrenalina, melhorando o humor ${ }^{(11)}$.

Deste modo, observa-se que é de suma relevância o incentivo à prática de atividades físicas no ambiente da universidade uma vez que os estudantes passam bastante tempo neste local. Logo, os profissionais de saúde poderão buscar parceria com outros órgãos/associações, bem como desenvolver ações com foco nas atividades desportivas e de lazer que estimulem a a participação dos estudantes.

Quanto ao consumo do tabaco, este foi considerado baixo visto que apenas 18 (5,4\%) estudantes eram tabagistas, portanto, um achado positivo que também foi encontrado em outras pesquisas com este público ${ }^{(18)}$. Já o uso de bebidas alcoólicas esteve presente em 121 estudantes universitários, constituindo um percentual significativo $(36,2 \%)$. 
Percebe-se que, atualmente, o uso e o abuso de álcool e/ou outras drogas lícitas e ilícitas têm aumentado progressivamente em todo o mundo, sobretudo entre os jovens. Portanto, consiste em um problema de saúde pública que tem necessitado de maior vigilância devido aos seus determinantes e condicionantes políticos, econômicos, culturais, ético-legais e tecnológicos, os quais se refletem no indivíduo, na família e na sociedade ${ }^{(20)}$.

Em relação a essa problemática, pesquisa realizada no Brasil em 2013, revelou a prevalência de $16,4 \%$ de consumo abusivo de álcool na população com 18 anos ou mais de idade ${ }^{(11)}$. No Timor-Leste, a prevalência de consumo atual de álcool (ingestão de bebidas nos últimos 30 dias) foi de 17,4\%, sendo a incidência bem maior nos homens (42,8\%). Entre aqueles que consumiram nos últimos 12 meses, 40\% eram consumidores mensais, cerca de $60 \%$ eram semanais e menos de $1 \%$ eram diários ${ }^{(21)}$.

No continente africano, a realidade também não difere do padrão global. No ano de 2004, o álcool foi o principal fator de risco para a deficiência ajustada de anos de vida (DALY) perdidos entre adolescentes e jovens africanos, na faixa etária de 15 a 24 anos, do sexo masculino ${ }^{(22)}$.

Destaca-se que o consumo de álcool e outras drogas aumenta a predisposição a acidentes automobilísticos, relações sexuais desprotegidas e transmissão do HIV, violência, distúrbios no padrão do sono, alterações dos hábitos alimentares, diminuição da percepção e elevação do estresse, além de ocasionar prejuízos acadêmicos ${ }^{(23)}$. Além disso, poderá também causar no indivíduo algumas alterações no humor, doenças cardiovasculares, doenças neurodegenerativas, gastrointestinais, hepáticas e mentais ${ }^{(24)}$.

Ressalta-se, ainda, que o uso de bebidas alcoólicas em estudantes universitários constitui uma problemática enfrentada pelos educadores e profissionais da saúde frente aos agravos de saúde e sociais que esse comportamento ocasiona ${ }^{(25)}$. Portanto, tal problema deverá ser abordado com maior ênfase na universidade, com ações de promoção de saúde (oficinas, cine debates) e utilização de outras estratégias capazes de reduzir esse hábito prejudicial à saúde.

Percebe-se, assim, que os hábitos de vida dos estudantes universitários de países lusófonos poderão acarretar maior risco para o desenvolvimento de doenças cardiovasculares uma vez que o fator hereditário está fortemente presente.

Neste âmbito, ainda é importante enfatizar que o continente africano enfrenta o crescimento acelerado das doenças cardiovasculares durante a sua transição econômica e pouco dispõe de recursos necessários no tocante à infraestrutura de saúde pública, quando comparada à dos países desenvolvidos. Além do mais, há diversas prioridades nacionais que competem com esses cuidados, como o estímulo ao crescimento econômico, às transformações sociais e políticas e os prejuízos decorrentes das doenças transmissíveis como o HIV/Aids ${ }^{(26)}$.

Apesar do relevante problema de saúde pública que são as DCV, encontraram-se poucos estudos envolvendo populações dos países lusófonos, sobretudo os da África, visto que geralmente não dispõem de recursos humanos e financeiros adequados. Logo, dados referentes às condições de saúde em geral e à identificação de fatores de risco cardiovascular podem ser subestimados.

Destarte, são necessárias ações voltadas para a promoção da saúde no meio acadêmico, de modo a sensibilizar este público para a importância da mudança do estilo de vida, com foco no incentivo às atividades desportivas como política de redução de danos, a qual visa controlar possíveis consequências negativas associadas ao consumo de substâncias psicoativas lícitas e ilícitas.

Considerando essa questão, esta pesquisa realizada no Brasil, com amostra de adultos universitários de diversos países lusófonos, é de notória importância, uma vez que os grupos participantes poderão ser replicadores, nos países de origem, das intervenções realizadas na universidade para a prevenção dos fatores de risco cardiovascular identificados. 


\section{CONCLUSÃO}

Este estudo, ao avaliar o risco cardiovascular dos estudantes universitários de países lusófonos, identificou os principais fatores de risco modificáveis e hereditários, de modo que pode contribuir para a comunidade acadêmica e para os profissionais de saúde por ter identificado os hábitos de vida de jovens que são prejudicais à saúde cardiovascular. Aponta-se que, apesar dos estudantes internacionais se encontrarem no Brasil, não houve viés.

Diante do diagnóstico situacional evidenciado nesta pesquisa, poderão ser desenvolvidas ações com foco na promoção da saúde, enfatizando a importância da atividade física e os prejuízos causados pelo uso do álcool, considerando os hábitos e a cultura de cada país.

Almeja-se que esta pesquisa proporcione aos profissionais de saúde e aos estudantes universitários ampliação de conhecimentos e de percepções na busca por uma prática que favoreça a saúde cardiovascular. Espera-se, ainda, que esta investigação possa subsidiar e servir de estímulo a outras nessa perspectiva, e que se apresente como alicerce para a construção de estratégias que favoreçam melhoria na qualidade do cuidado deste grupo.

\section{REFERÊNCIAS}

1. World Health Organization (WHO).Cardiovascular diseases (CVDs).[Internet] WHO; 2017 [acesso em 02 agosto 2017]. Disponivel: http://www.who.int/mediacentre/factsheets/fs317/en/.

2. World Health Organization (WHO).Cardiovascular diseases (CVDs). [internet] WHO; 2017 [acesso em 13 ago 2017]. Disponível:http://www.afro.who.int/pt/node/521.

3. Mansur AP, Favarato D. Mortalidade por Doenças Cardiovasculares em Mulheres e Homens nas cinco Regiões do Brasil, 1980-2012. Arq.bras.cardiol. [Internet] 2016 [acesso em 01 ago 2017]. Disponível: http://dx.doi.org/10.5935/ abc.20160102.

4. Chimhundu C, de Jager K, Douglas T. Focus areas of cardiovascular medical device research in South Africa. S. Afr. Med. J. [Internet]2016;106(1) [acesso em31 jul 2017].Disponível: http://dx.doi.org/10.7196/SAMJ.2016.v106i1.10166.

5. de Brito BB, Leal JDV, Formiga LMF, Frota KMG, da Silva ARV, Lima LHO. Cardiovascular Diseases: Risk Factors in Adolescents. Cogitare Enferm. [Internet] 2016;21(2) [acesso em 31 jul 2017]. Disponível: http://dx.doi.org/10.5380/ ce.v21i2.41848.

6. Morales G, Guillen-Grima F, Muñoz S, Belmar C, Schifferli I, Muñoz Aet al. Factores de riesgo cardiovascular enuniversitarios de primer y terceraño. Rev. Méd. Chile.[Internet]2017;145(3) [acesso em 03 ago 2017]. Disponível: http://dx.doi.org/10.4067/S0034-98872017000300003.

7. Bonotto GM, Mendoza-Sassi RA, Susin LRO. Conhecimento dos fatores de risco modificáveis para doença cardiovascular entre mulheres e seus fatores associados: um estudo de base populacional. Ciênc. saúde coletiva. [Internet]2016;21(1) [acesso em 02 ago 2017]. Disponível: http://dx.doi.org/10.1590/1413-81232015211.07232015.

8. Shawar SM. Al-Bati NA, Ali-Mahameed A, Nagalla S, Obeidat M. Hypercholesterolemia among apparently healthy university students. Oman Med. J. [Internet] 2012;27(4) [acesso em 30 jul 2017]. Disponível: http://dx.doi.org/10.5001/ omj.2012.69.

9. Arts J, Fernandez ML, Lofgren IE. Coronary Heart Disease Risk Factors in College Students. Adv Nutr. [Internet] 2014;5(2) [acesso em 30 jul 2017]. Disponível: http://dx.doi.org/10.3945/an.113.005447.

10. Sociedade Brasileira de Cardiologia (SBC). $7^{a}$ Diretriz Brasileira de Hipertensão Arterial. Arq.bras.cardiol. [Internet] 2016;107(3Suppl.3) [acesso em 13 jul 2017]. Disponível: http://publicacoes.cardiol.br/2014/diretrizes/2016/05_HIPERTENSAO_ARTERIAL.pdf. 
11. Bermúdez HFC, Collazos JEM, Fonseca AA. Evaluación de razones de prevalencia para sedentarismo y factores de riesgoenun grupo de estudiantes universitarios. Chía - Columbia. Enferm. glob. [Internet] 2014;13(2) [acesso em 23 jul 2017]. Disponível: http://dx.doi.org/10.6018/eglobal.13.2.172951.

12. Ministério da Saúde (BR). Secretaria de Vigilância em Saúde. Departamento de Vigilância de Doenças e Agravos não Transmissíveis e Promoção da Saúde. Vigitel Brasil 2013: vigilância de fatores de risco e proteção para doenças crônicas por inquérito telefônico. [internet] Brasília: Ministério da Saúde; 2014 [acesso em 12jul 2017]. Disponível: http://bvsms. saude.gov.br/bvs/publicacoes/vigitel_brasil_2013.pdf.

13. World Health Organization (WHO). Who Report on the Global Tobacco Epidemic. Monitoring tobacco use and prevention policies.2017. [Internet] WHO; 2017 [acesso em 17 ago 2017]. Disponível: http://apps.who.int/iris/ bitstream/10665/255874 /1/9789241512824-eng.pdf?ua=1\&ua=1.

14. Cruz-Sánchez E, Orosio-Méndez M, Cruz-Ramírez T, Bernardino-García A, Vásquez-Domínguez L, GalindoPalma $\mathrm{N}$ et.al. Factores de riesgo cardiovascular enestudiantes de enfermería de una universidad pública. Enferm. univ. [Internet] 2016;13(4) [acesso em 07 ago 2017]. Disponível: https://doi.org/10.1016/j.reu.2016.09.004.

15. deArruda GO, Santos AL, Teston EF, Cecilio HPM, Radovanovic CAT, Marcon SS. Association between self-reported health and sociodemographic characteristics with cardiovascular diseases in adults. Rev. Esc.Enferm. USP. [Internet]2015;49(1) [acesso em 03 ago 2017]. Disponível: http://dx.doi.org/10.1590/S0080-623420150000100008.

16. Arnold TJ,Schweitzer A,Hoffman HJ,Onyewu C,Hurtado ME,Hoffman EPet al. Neck and waist circumference biomarkers of cardiovascular risk in a cohort of predominantly African-American college students: a preliminary study. JAcadNutrDiet. [Internet]2014;114(1) [acesso em 15 jul 2017]. Disponível: http://dx.doi.org/10.1016/j.jand.2013.07.005.

17. Stein R, Trujillo JP, da Silveira AD, Júnior AL, Iglesias LM. Avaliação Genética, Estudo Familiar e Exercício Arq.Bras. Cardiol. [Internet] 2017;108(3) [acesso em 27 jul 2017]. Disponível: http://dx.doi.org/10.5935/abc.20170015.

18. deCarvalho CA, Fonseca PCA, Barbosa JB, Machado SP, dos Santos AM, da Silva AAM. Associação entre fatores de risco cardiovascular e indicadores antropométricos de obesidade em universitários de São Luís, Maranhão, Brasil. Ciênc. saúde coletiva. [Internet] 2015;20(2) [acesso em 22 jul 2017]. Disponível: http://dx.doi.org/10.1590/141381232015202.02342014 .

19. Dázio EMR, Zago MMF, Fava SMCL. Usode álcool e outras drogas entre universitários do sexo masculino e seus significados.Rev. esc.enferm. USP. [Internet] 2016;50(5) [acesso em 28 jul 2017]. Disponível: http://dx.doi.org/10.1590/ S0080-623420160000600011.

20. World Health Organization (WHO).Global Status Report on Alcohol and Health 2014. [internet] WHO; 2014 [acesso em 05 ago 2017]. Disponível: http://apps.who.int/iris/bitstream/10665/112736/1/9789240692763_eng.pdf.

21. World Health Organization (WHO). National Survey for Non-Communicable Disease Risk Factors and Injuries: Using WHO STEPS approach in Timor-Leste - 2014. [Internet] WHO; 2014 [acesso em 22 jul 2017]. Disponível: http:// apps.who.int/iris/bitstream/10665/204350/5/B5224.pdf.

22. Zeferino MT, Hamilton H, Brands B, Wright MGM, Cumsille F, Khenti A. Consumo de drogas entre estudantes universitários: família, espiritualidade e entretenimento moderando a influência dos pares. Texto contexto - enferm. [Internet]2015;24(n.esp) [acesso em 01 ago 2017]. Disponível: http://dx.doi.org/10.1590/0104-07072015001150014.

23. Nunes JM, Campolina LR, Vieira MA, Caldeira AP.Consumo de bebidas alcoólicas e prática do bingedrinking entre acadêmicos da área da saúde. Rev.psiquiatr.clin. [Internet] 2012;39(3) [acesso em 12 jul 2017]. Disponível:http://dx. doi.org/10.1590/S0101-60832012000300005.

24. Cichocki M, Fernandes KP, Castro-Alves DC, Gomes MVM. Atividade Física e Modulação do Risco Cardiovascular. Rev. BrasMed Esporte. [Internet] 2017;23(1) [acesso em 25 jul 2017]. Disponível: http://dx.doi.org/10.1590/1517869220172301159475.

25. Maciel MED, Vargas D. Consumo de álcool entre estudantes de enfermagem. Rev Fund Care Online. [Internet] 
2017;9(1). [acesso em 01 ago 2017]. Disponível: http://dx.doi.org/10.9789/2175-5361.2017.v9i1.64-70.

26. Capingana DP. Fatores de risco cardiovascular em diferentes níveis socioeconômicos de servidores públicos da universidade Agostinho Neto, Luanda, Angola [tese].Vitória (ES): Universidade Federal do Espírito Santo; 2012. Disponível: http://portais4.ufes.br/posgrad/teses/tese_5824_Tese\%20Daniel\%20Capingana.pdf. 